\title{
Réparer le monde : excès, reste et innovation
}

Frédéric Joulian, Yann Philippe Tastevin et Jamie Furniss

\section{OpenEdition}

Journals

Édition électronique

URL : https://journals.openedition.org/tc/7772

DOI : $10.4000 /$ tc. 7772

ISSN : 1952-420X

\section{Éditeur}

Éditions de l'EHESS

\section{Édition imprimée}

Date de publication : 31 octobre 2016

Pagination : 14-27

ISBN : 9782713225291

ISSN : 0248-6016

\section{Référence électronique}

Frédéric Joulian, Yann Philippe Tastevin et Jamie Furniss, « Réparer le monde : excès, reste et innovation », Techniques \& Culture [En ligne], 65-66 | 2016, mis en ligne le 31 octobre 2019, consulté le 29 septembre 2022. URL : http://journals.openedition.org/tc/7772 ; DOI : https://doi.org/10.4000/tc. 7772 


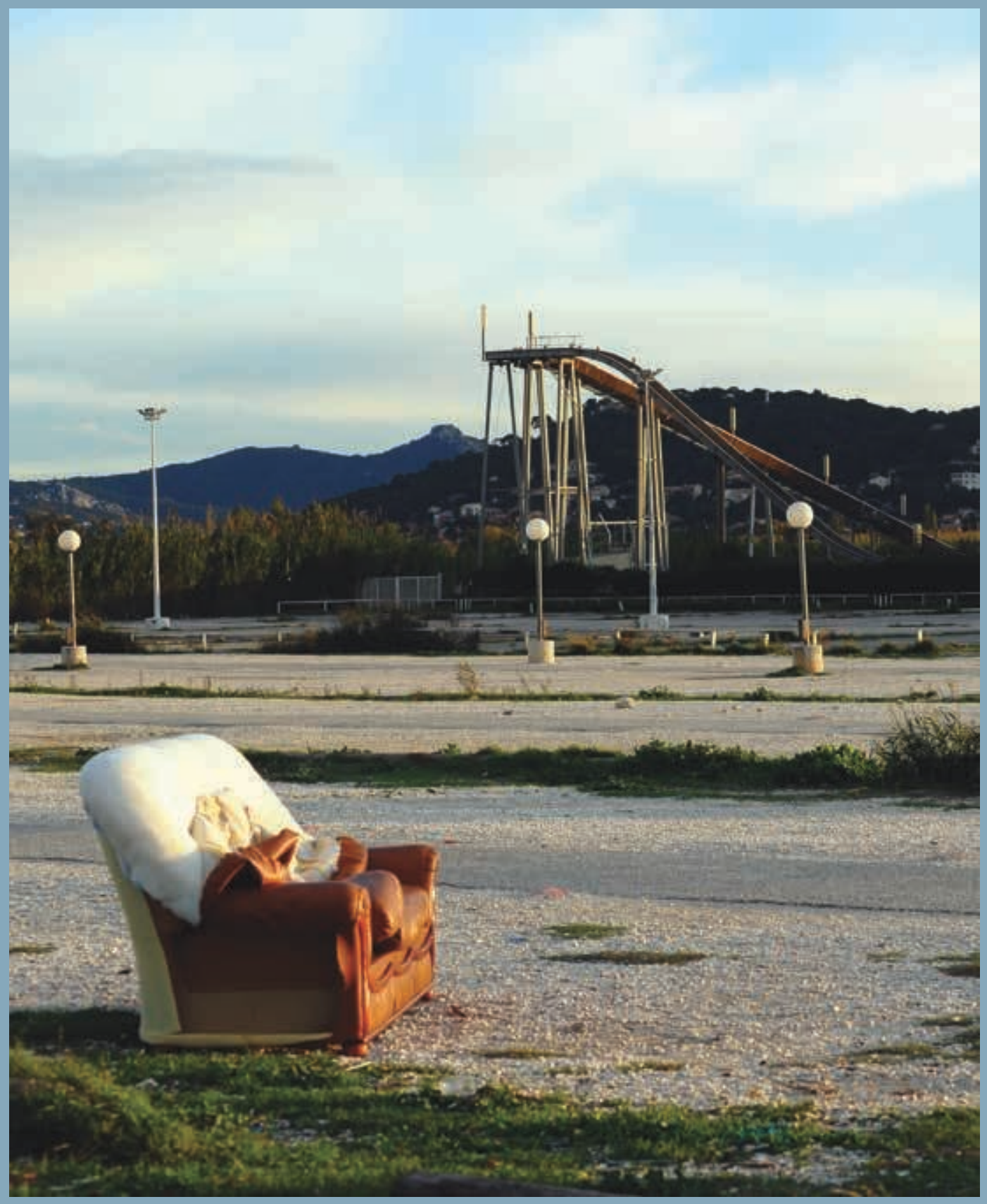




\section{Réparer le monde : excès, reste et innovation}

L'ouvrage que vous venez d'ouvrir est l'un des plus volumineux de l'histoire, cette année quarantenaire, de la revue TechniquesÉCulture. Il concrétise un projet mené sur plus de cinq ans mobilisant divers acteurs et partenariats scientifiques (CNRS, MUCEM, EHESS) sur différents temps, individuels et collectifs, de réflexion et de recherche. Par sa diversité, il embrasse un immense champ d'étude que nous avons tenté de cerner de la façon la plus englobante possible. L'histoire de ce volume trouve son origine dans le séminaire «Out of culture: la société par ses restes» (Joulian \& Jeanjean 2014) dans lequel, de 2011 à 2013, nous avons interrogé la question de l'universalité de la culture au regard des objets et sujets délaissés de nos sociétés. Pour un collectif humain peut-il y avoir réellement des choses qui soient «hors culture », qui ne fassent pas sens? Qui soient délaissées, impensées ou refoulées telles certaines matières ou idées? Telles étaient nos interrogations originelles.

Le ou les restes, pris dans leurs différentes histoires et généalogies, ou dans des comparaisons raisonnées, s'avèrent de fait essentiels pour révéler les points aveugles de la recherche en sciences humaines, que ce soit à l'égard des sociétés de consommation, des primates «non humains » dotés désormais d'objets et de traditions, ou, à l'inverse, d'hommes préhistoriques «pré-culturels» ou de «natures» pensées autonomes de la sphère anthropique. Alliant cette réflexion d'anthropologie générale à une approche politique des enjeux sociaux, vus d'en bas, vus par les personnes en charge de gérer les reflux de nos digestions et activités, ce séminaire fut l'occasion d'explorer de façon systématique les différentes instances impliquées dans la gestion des matières ou des artéfacts, périssables ou pérennes, peu ou fortement transformés. Les objets interrogés tout au long de ce parcours vont des minerais rares aux excréments quotidiens, des résidus de la mine au cadavre pris comme un déchet, d'ailleurs déjà questionné dans un numéro précédent, «Le cadavre en procès », dans les effets qu'il produit sur les vivants qui le côtoient (Guy, Jeanjean \& Richier 2013).

Nous étoffant des contributions de Yann-Philippe Tastevin (2012) et de Jamie Furniss (2012) nous associâmes TechniquesÉCulture au projet d'exposition «Vies d'ordures» sur l'économie des déchets 
1. Artéfacts naturels. Certains non-humains, ici des chimpanzés, plient, ploient, croisent des branches sur des fourches d'arbres sans les casser, les tapissent de feuilles: ils aménagent leurs «nids» plus qu'ils ne les «fabriquent».

La longue histoire humaine, celle des chasseurs-cueilleurs s'est, jusqu'à l'Holocène, déroulée d'une façon peu vulnérante; la rupture moderne n'en est que plus scandaleuse. (Nyama-Yara, Guinée 2005).

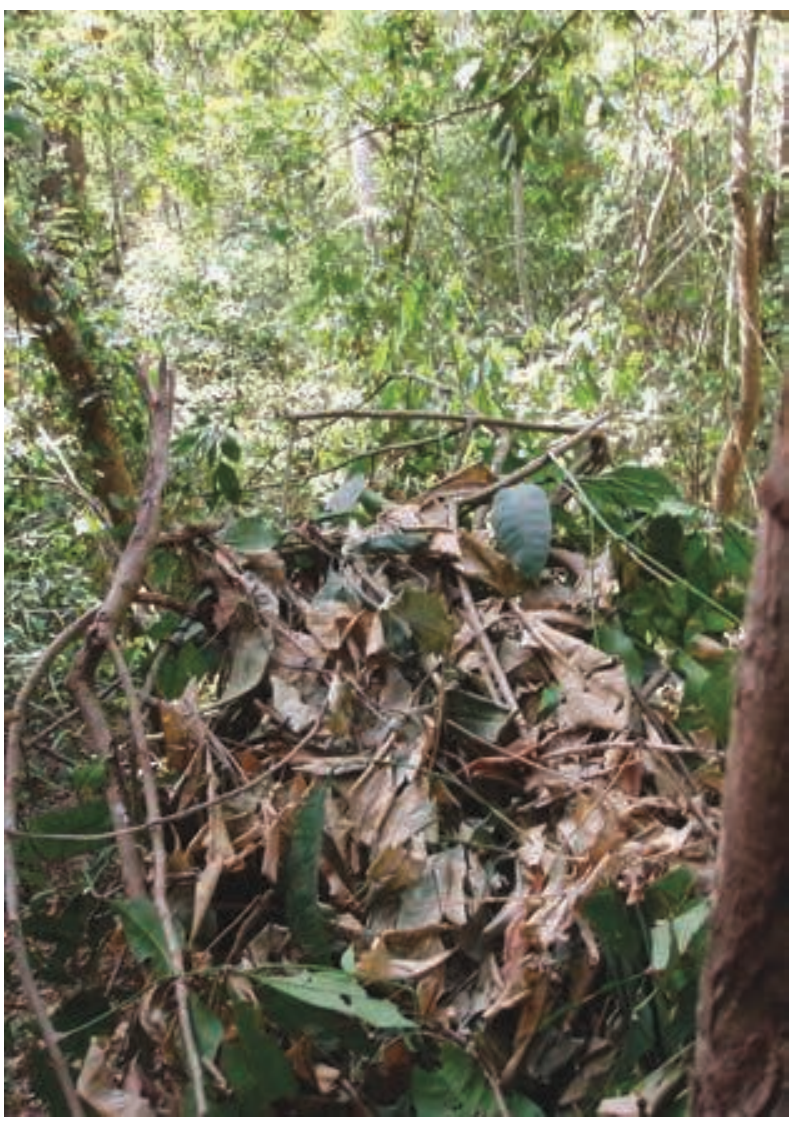

et du recyclage en Méditerranée, lancé par Denis Chevallier au MUCEM (prévue de mars à septembre 2017): les deux projets, éditorial d'un côté, muséographique de l'autre, se nourrissant mutuellement. Nous tenons ici à remercier chaleureusement tant les partenaires de la revue (P.-O. Dittmar, M.-L. Rauzy, D. Bally, S. Zingraff, J.-B.Pla) que du Musée (D. Chevallier, A. Fanlo, M.-C. Calafat, L. Lane) qui ont généreusement contribué à ce rapprochement. Lattention conjointe des uns et des autres, tant aux dimensions scientifiques que publiques, mérite d'être saluée.

\section{Heuristique du reste}

Le lancement de cet ouvrage ne débuta cependant qu'avec l'argumentaire et l'appel à contributions, qui comprennent une typologie analytique (Restes «irréductibles», Restes «réutilisés», Restes « retransformés », Restes «fantômes »,

Restes «excédentaires» cf. tc.revues.org/6987) et qui constituent autant de pistes ouvertes pour penser ensemble les matérialités, les différents régimes de temporalité, de valeur et de visibilité des restes et des déchets. L'idée de cet appel (diffusé assez largement à l'étranger) était de faire apparaître la diversité des recherches en cours sur les restes et les excès, mais aussi la variété des réponses techniques ou sociales qui leur sont faites. Nous tentâmes également de faire attention à ne pas trop canaliser la formulation de l'argumentaire sous les expressions les plus en vogue à l'heure actuelle (celles de «deuxième vie d'objets», mais également celles «d'Anthropocène», de «durabilité», « d'économie circulaire» ou de «résilience ») qui risquaient de polariser par trop les réponses dans un sens ou dans un autre, de dramatiser les problématisations, d'empêcher une appréhension mesurée des phénomènes, ou de circonscrire le champ d'étude de façon trop étroite. Nous proposons donc une réflexion générale sur la notion de reste articulée à celle de déchet afin que les catégories des rejets soient laissées les plus ouvertes possibles. À l'instar des «Discard Studies» (discardstudies.com) nous estimons que la matérialité de nos déchets et leurs significations font partie de systèmes techniques, socioculturels et économiques plus larges. Le mot « reste» ne devrait cependant pas être considéré comme la traduction 


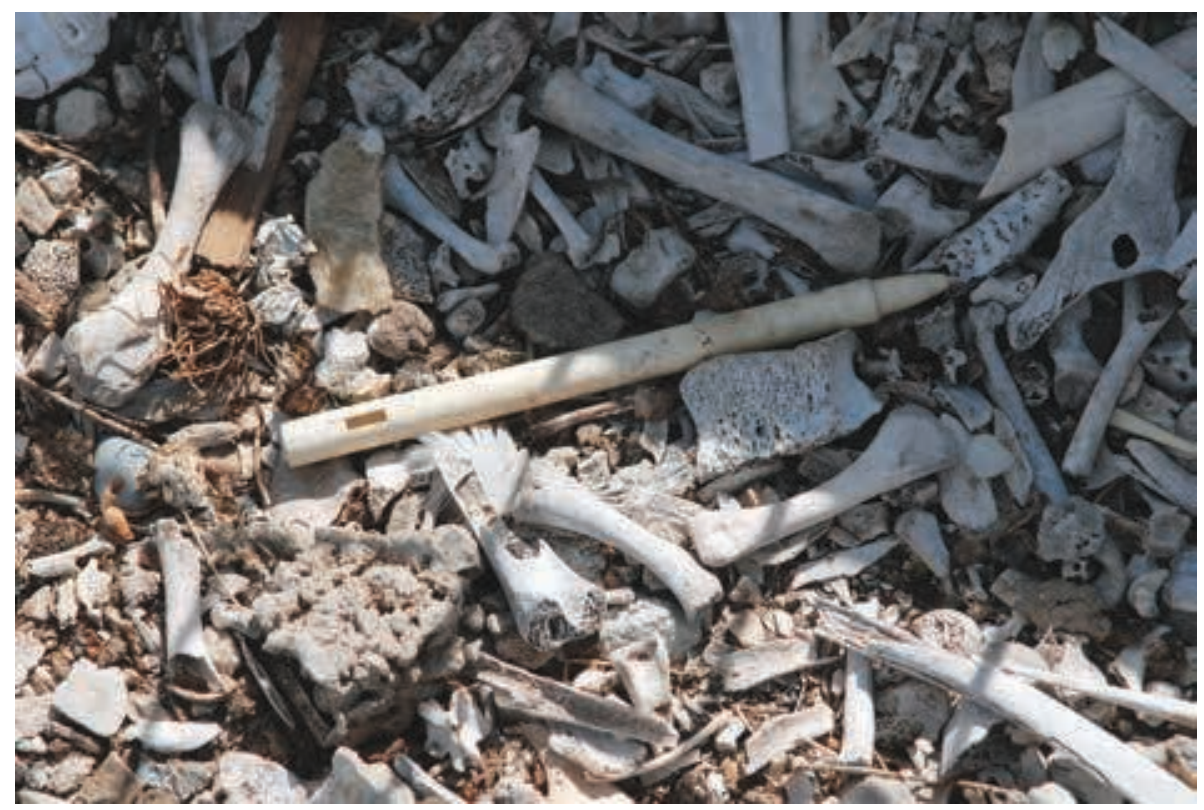

2. Stylo de goélands. Les goélands, - oiseaux extrêmement intelligents et opportunistes, colonisent les toits terrasses de Marseille. De leurs raids sur la ville ou le littoral, ils rapportent différents aliments (poissons, coquillages, jeunes pigeons tués ou carcasses de poulets cuits récupérés dans les poubelles ou sur les marchés) mais aussi d'étranges objets avec lesquels ils jouent et apprennent. Ici, un stylo de la DGA, léger comme une diaphyse osseuse d'oiseau, a été transporté. A-t-il leurré un jeune? D'où vient-il? De la lointaine direction générale de l'Armement ou de la proche direction générale des Assurances? Cet exemple illustre en revanche pour nous l'importance de «dés-anthropocentrer» les propos et de bien saisir la multiplicité des facteurs et vecteurs à l'œuvre dans la dispersion et l'impact des déchets dans tous les milieux, qu'ils soient faiblement ou fortement anthropisés.

française de «discard» car la dimension intentionnelle du terme anglais - qui signifie à la fois l'action de dessaisir et l'objet qui est dessaisi- recouvre mal les dimensions non intentionnelles et invisibles que nous souhaitons inclure. Notre tâche consiste à interroger la façon dont les déchets adviennent et jouent sur les humains et non-humains. Mais comment aborder ensemble les dimensions thermodynamiques, économiques, techniques ou ontologiques, mémorielles et symboliques, si ce n'est à l'aide de voix multiples et accordées? Puisque les restes sont transverses, multiples, ils appellent à une confrontation et à une interrogation collective et interdisciplinaire.

Enfin, de façon affirmée, nous postulons que les restes et leur traitement ont une valeur heuristique originale pour les sciences sociales. Les textes proposés ici réunissent des contributions issues de diverses disciplines provenant de terrains proches et éloignés où le reste est envisagé non seulement comme « revers de la production» mais aussi comme un objet bon à penser car cristallisant les dimensions pratiques et symboliques. En nous intéressant tant aux matières et matériaux qu'aux objets et produits finis, nous tentons de surmonter les limites de l'anthropologie des objets ou celle de la consommation. Par les formes dégradées, nous renversons les filières et chaînes opératoires, allons de l'aval vers l'amont, «bouclons» et réintroduisons les circulations des savoirs, des processus et du travail.

\section{Temporalités des restes}

La rudologie (du latin rudus, décombres) désigne l'analyse raisonnée et scientifique des déchets en tant que témoins de sociétés données et de leurs inscriptions spatiales. On doit ce terme en 


\section{Le Caire.}

Machines/machin: les machines sont des choses dont on connaît les mécaniques internes et que l'on peut donc réparer, adapter, bidouiller...; les machins sont des choses dont on ignore le fonctionnement, ça marche mais on ne sait pas comment, d'où l'impossibilité de les réparer. Le rapport humain, avec la modernité est passé des machines aux machins..

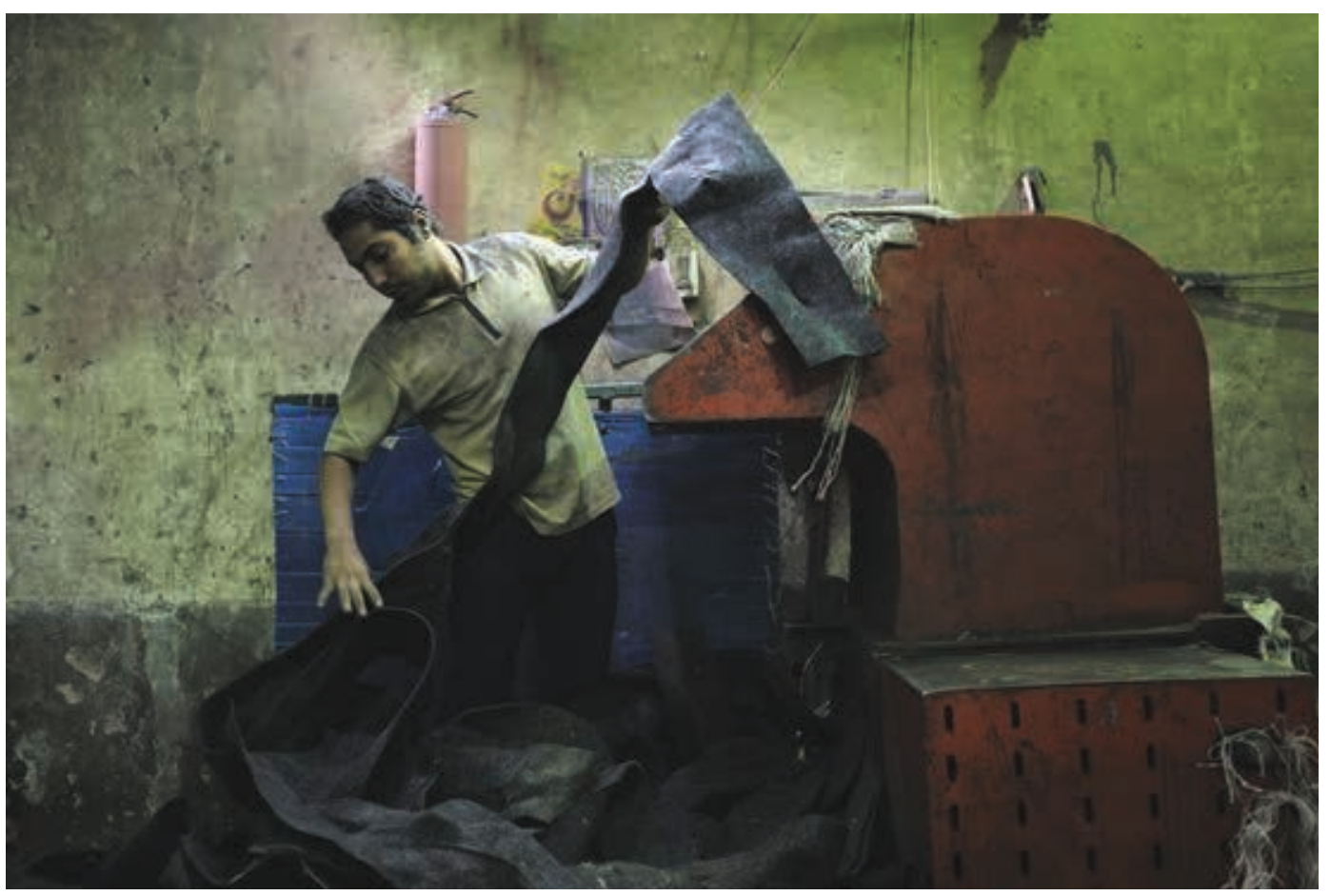

France à Jean Gouhier (1972). Aux États-Unis, William Rathje a fait de l'archéologie des poubelles une dimension à part entière de la sociologie de la consommation nord-américaine (1980) bien avant que les géologues tenants de l'Anthropocène prennent les restes anthropiques comme les indicateurs du grand changement du Pléistocène à «l'Anthropocène »-l'Holocène disparaissant dans le même mouvement! (Steffen et al. 2011, Zalasiewicz et al. 2012). L'Anthropocène fournit un cadre de réflexion stimulant pour penser à de nouveaux frais la nature des déchets; leur existence est solidaire de boucles de rétroaction biochimiques d'échelle planétaire qui déterminent en grande partie les temporalités d'assimilation dont les durées de persistance se situent souvent dans le «temps profond», à des échelles d'effets et de temps en inadéquation avec la vie humaine.

Sans compter le rôle des restes dans les débats émergents sur le «temps profond» des ères géologiques (Irvine 2014), les approches historiques ont quant à elles mis en lumière des variations importantes dans la relation des êtres humains à leurs résidus, excreta et surplus (Barles 2005, Strasser 2000, Zimring 2005). Sabine Barles rappelle à juste titre qu'en dépit de l'ardeur des idées hygiénistes et modernisatrices, c'est finalement l'invention de techniques permettant de fabriquer le papier à base de pulpe de cellulose qui marquera la disparation lente du chiffonnage à Paris, vers le milieu du xxe siècle. Gabrys (2013) propose une « histoire naturelle» des appareils électroniques et montre comment les technologies dématérialisées et virtuelles continuent à avoir une empreinte écologique considérable. Le travail d'historicisation est donc indispensable, ne serait-ce que pour 
lutter contre le fantasme de sociétés pré-industrielles ou post-modernes sans déchet. Plutôt que rêver une hypothétique absence de déchet, passé ou à venir, on gagne à penser non seulement ce que les humains font des déchets, mais ce que les déchets font aux humains.

Ce à quoi nous avons affaire, ce n'est pas tant la constitution d'un environnement de l'homme, fut-il délétère, que celle de nouveaux milieux, de nouveaux ordres territoriaux soumis à la puissance des restes, qui génèrent des intrications inédites entre humains et non-humains. Le sol, l'eau, l'air, les corps se défont de la neutralité et gardent «en mémoire» les traces de la chimie industrielle, des pollutions agricoles (Duperrex 2015). En dépit des efforts d'invisibilisation, les paysages, les instruments de mesure (physiques et sociaux) ou les espèces «sentinelles» permettent de rendre compte de matières et de dangers parfois difficilement décelables par nos appareils perceptifs, qu'il s'agisse des pollutions atmosphériques, du plastique dans les mers ou de la radioactivité. L'enjeu anthropologique est alors de donner à voir et à comprendre cette transformation ou dégradation biosocio-techno-symbolique qui se joue, non dans des milieux «purs» (ceux de la culture ou ceux de la nature), mais dans des milieux « hétérogènes » (faits de machines fonctionnantes, de restes cassés, de «machins»), ainsi qu'avec les produits et sous-produits nocifs de la surconsommation. Rappelons à ce propos les premières grandes alarmes de la fin des années 1960-1970 avec les travaux du Club de Rome, la première crise pétrolière de 1973-1974 et des réponses alternatives (l'énergie solaire ou l'agriculture durable) qui leur furent faites (Schumacher 1973, Illich 1973, Borasi \& Zardini 2007).

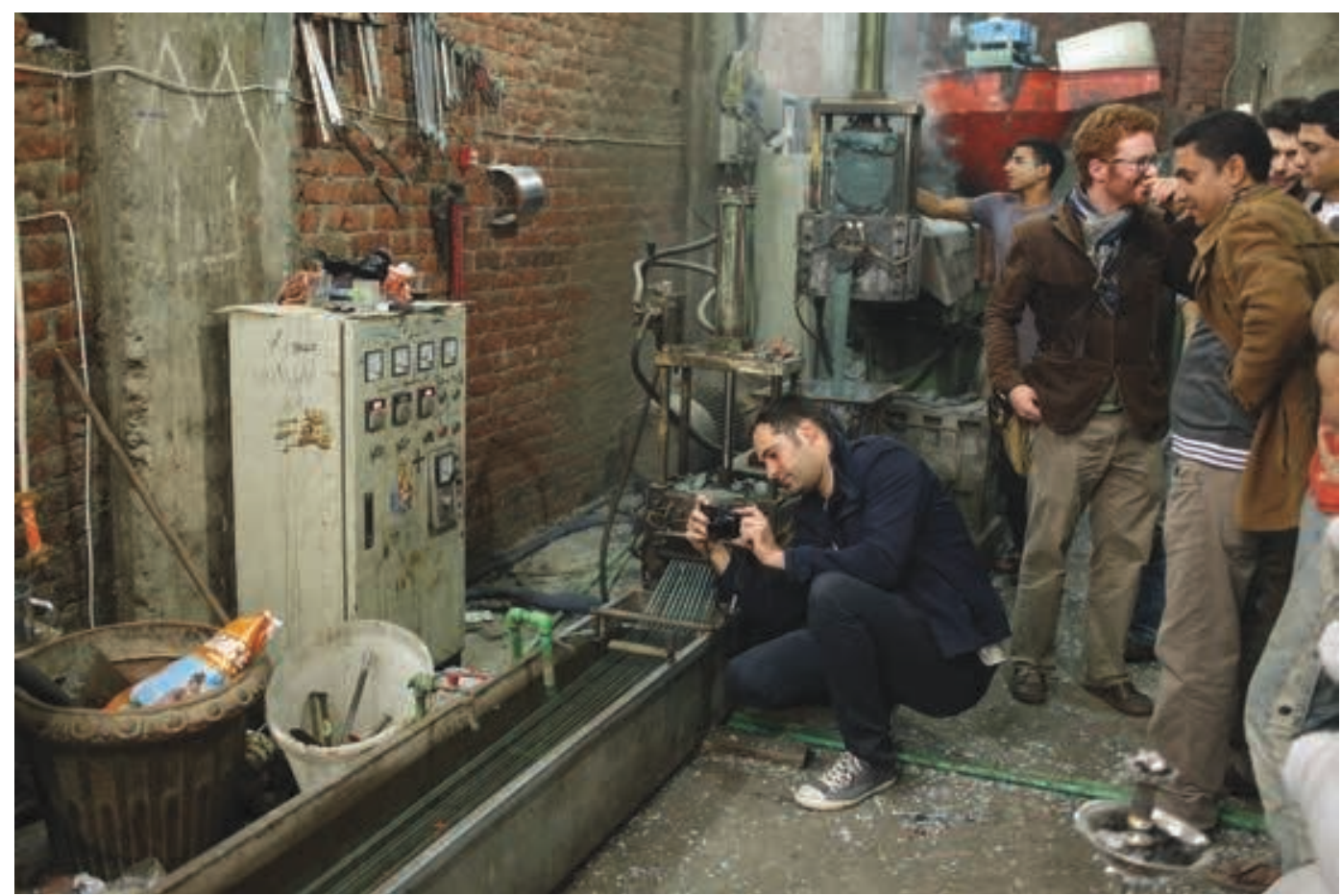

Ce que nos enquêtes observent est un processus inverse: à partir des machins, des hommes et des femmes fabriquent des machines (et produisent des matières premières). Au Caire ou en Tunisie on observe des processus où l'on repasse des machins aux machines; des savoirs expérimentaux qui nécessitent un hors monde (un laboratoire) aux gestes pragmatiques des autodidactes par expérience sensible de la matière. 


\section{O ANS DE CREDIT ÉNORME POUR SE PAYER CETTE SALOPERIE}

Exemple de financement: Apport personanel $64.000 \mathrm{~F} * *$ 5 ans à $2380 \mathrm{~F} / \mathrm{mois}$. 10 ans aे $2760 \mathrm{~F} / \mathrm{mois}$.
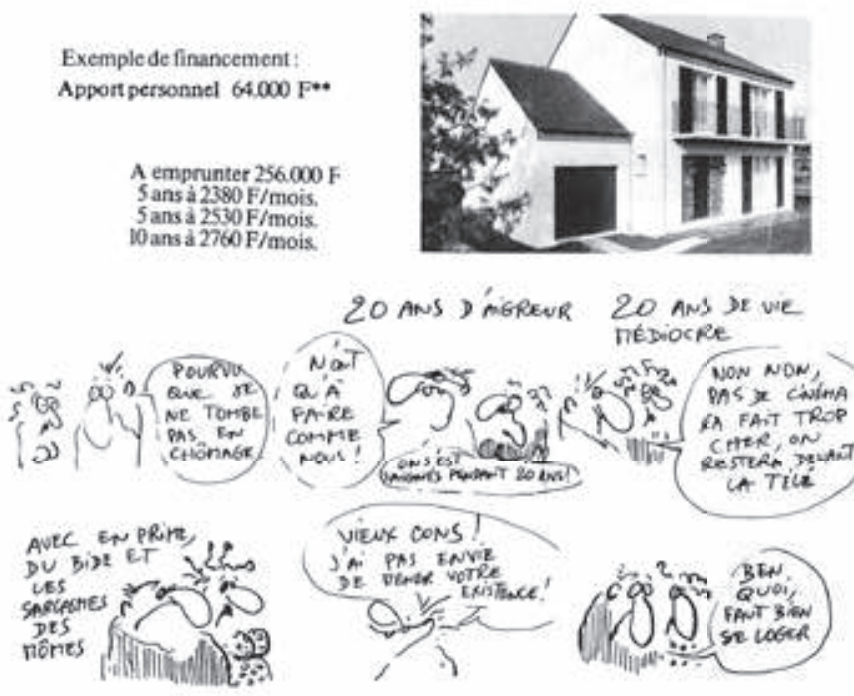

\section{architecture marginale auX U.S.A}

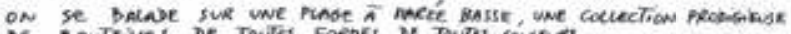

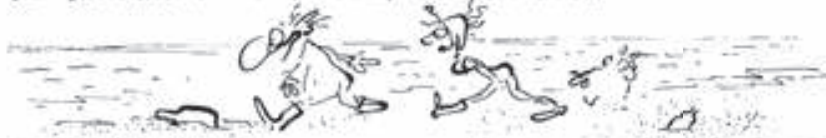

ON RATASSE UES Bouteicus.

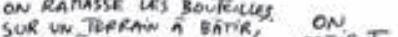

ON 3 ATTIT

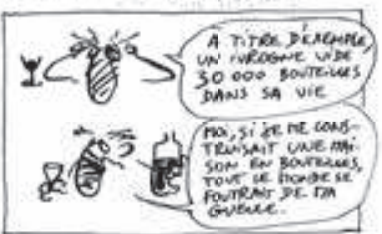

De la fin des années soixante au début des années quatre-vingt et après le premier choc pétrolier de 1973 (Borasi, Zardini 2007), les scien tifiques et les politiques (Club de Rome, Groupe des Dix; Chamak 200o) posèrent les bases d'une réflexion et interpellation sur le devenir de la pla nète. D'autres utopistes, plus pragmatiques, tel le dessinateur Reiser, s'engagèrent dans l'énergie solaire et le développement durable. Ce volume est comme un fruit tardif de ces réflexions et engagements.

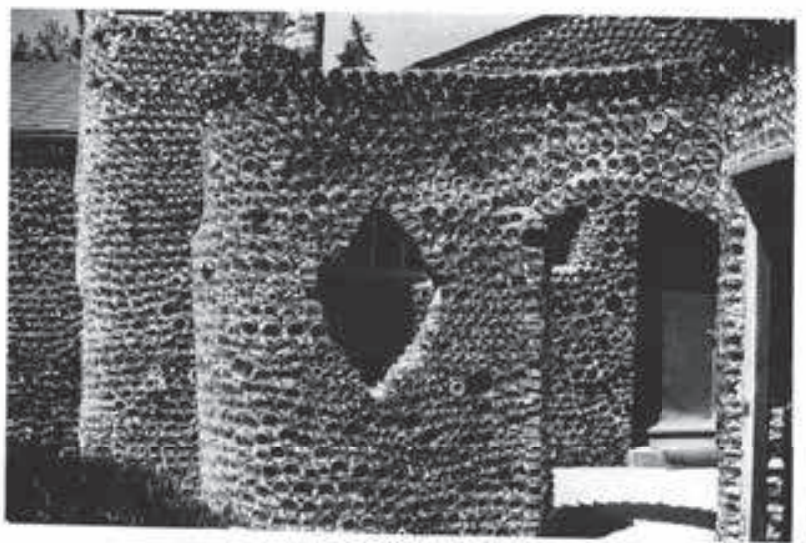

emprunter $256000 \mathrm{~F}$

BALNDE - vOUS EN FOREt, RATASSEz DES sours de kois, vous constrivitere \&A

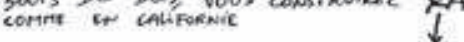

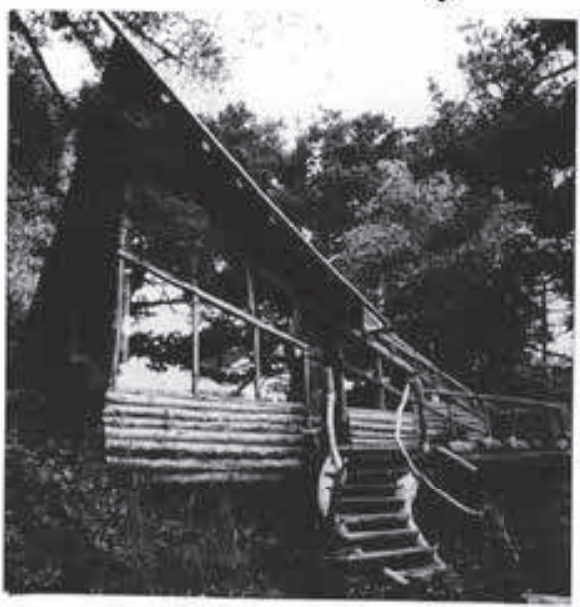

non: varee

DANS VES CASSES DE VOITURES, DÉCOUPEZ ES CAPOTS, Vous Construviter AA?

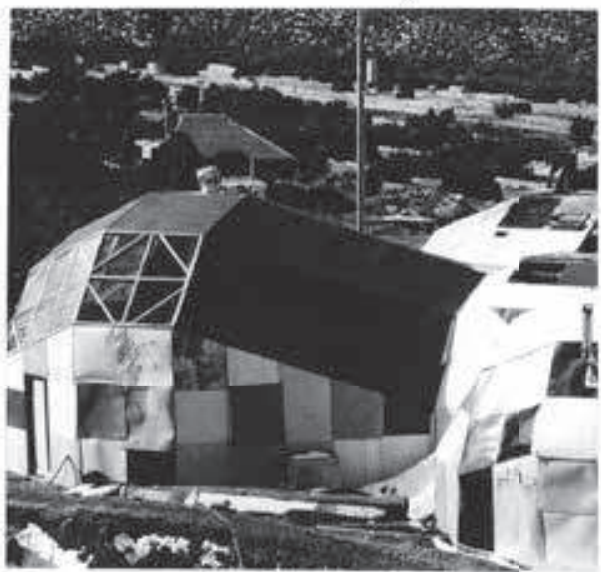

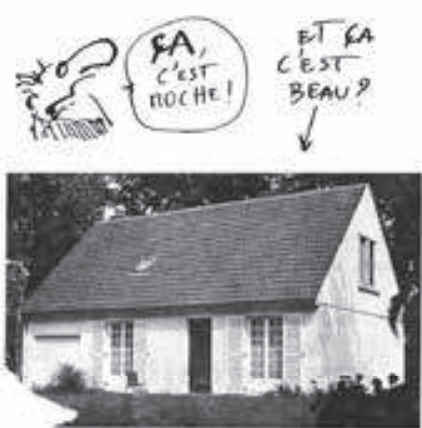

CARAvEuE F a. Torraln 060 m2 20000 F

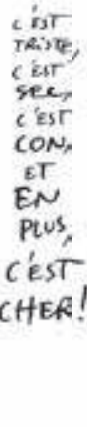




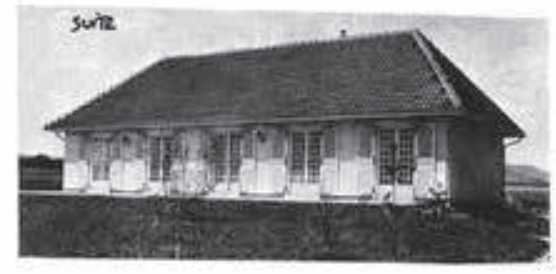

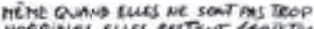

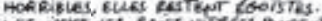

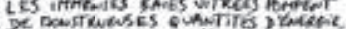
osugenut a covirivice cemiraces

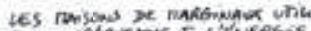

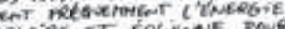

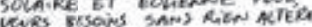
AKTTOUK
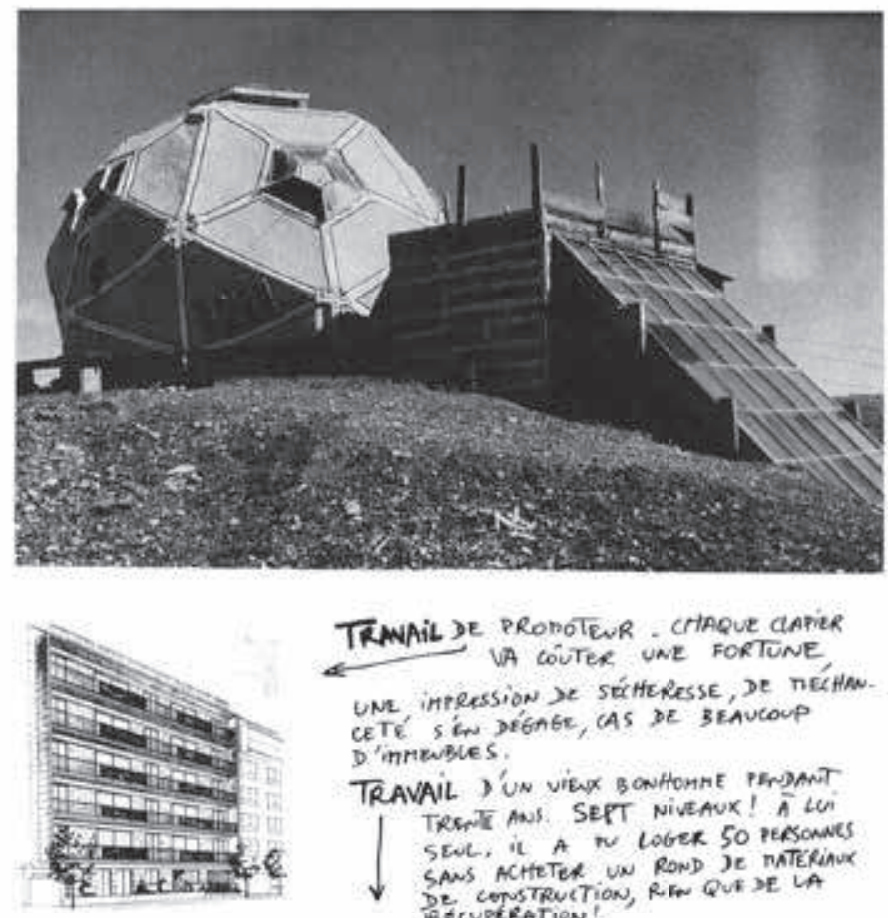

TRWAIL DE PRONOTEWR. CHAQUE CAATER I- VA WÓUTER UAE FORTUNE, UNe itrRession de SÉ́tERESSE, DE TELHAN. UNE, ATRESSION SE CAS DE BEAUCOUP D. in rewbles.

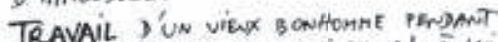
TRST AOUS SEPT NIVEAUX! A LCA

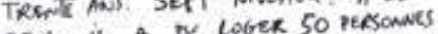

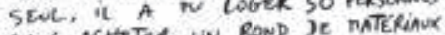
SANS ACHETER UN ROND SUE $Y$ E LA De construction
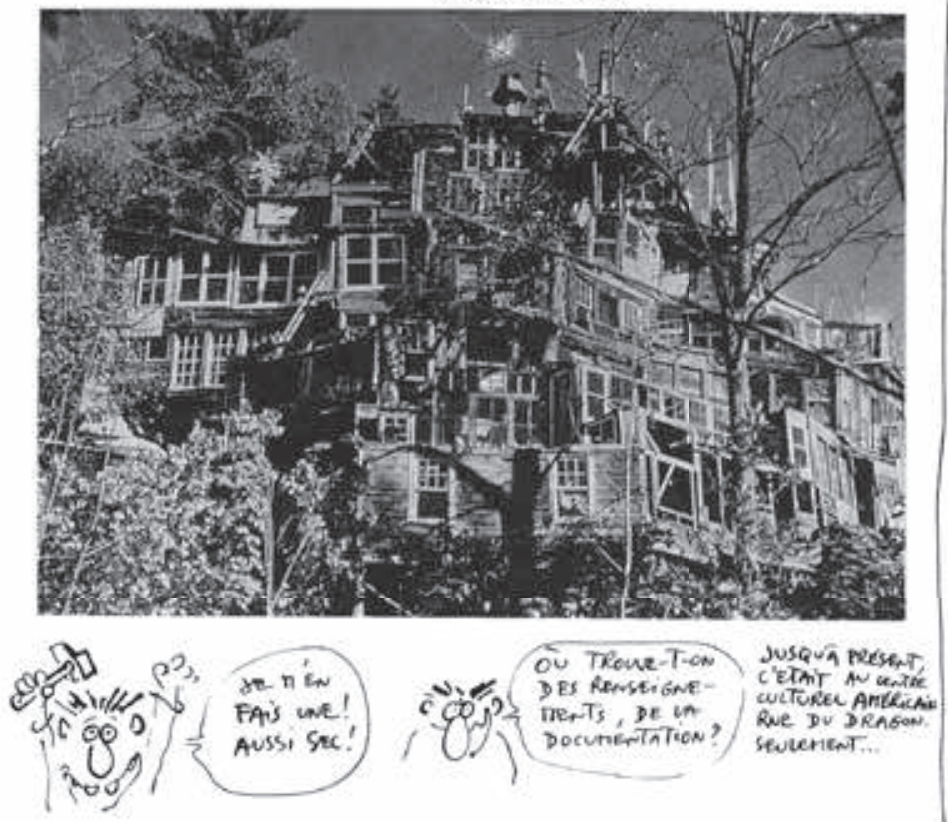

SEULEMENT... En Pus of Bentos DE DABSons, Y'AUAT DES vivRES SUSPECTS, DES Citations pólitiques... Ie marcuse eartre autres

* La fonction politique de lécologie est Facle a neutraliser, cLue pent etre tork. NÉE Á la GLRification DV SYSTËRE, ET POURTANT, IS FANT COMBATTRE 20 , ET RAWTENANT LA POLIUTíON PHYSIQUE PRATIQUÉE PAR IE SHTEME, TOUT COMEE SA POLLUTION HENTALE POUR Anener l'écologie au point OV eULe n'est PLUS COMPATISLE AVEC LES STRUCTURES CAPITA. WSTES IL FAUT D'ABORD DEUECOPPER UA CAMPASEE ÉCOOGIQUE A L'NTTRRIEUR DE CES STRUTERES》

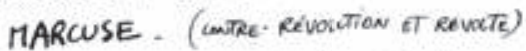

ET puis DES PHotos DE nARGimavX $\hat{A}$ Poit. ALORS LAMBASSADE US A RÉAG C ATHERIQUE QUT

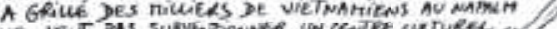

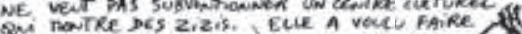

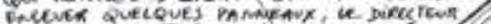

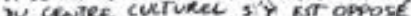
A PRTFERE QUE I'EXPO Frthe. FinAlenent, rlle. SAR OUVRTE DE MaNEAU AU CCi 107 RUE De Rivoli

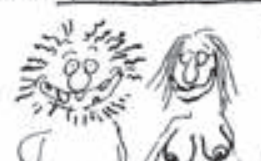

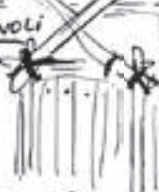

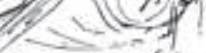
तiा मेंती

\section{CENSURER UNE EXPOSITION PAREILLE... L'EXPO Qui NaUs A TATT oublick un motient}
L'ATÉRIQVUE
DES MONSTRES
DE LA TAFFIA
DES ROBOT
ASSASSINS
DES PRESTENT
VEREX

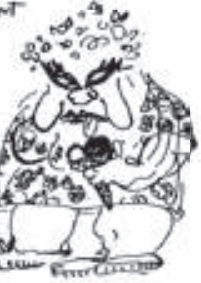
Des Trusts REQvin', ov RACISHE.

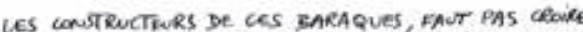

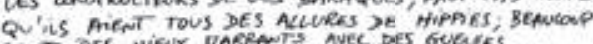

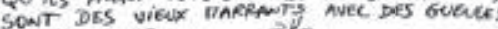
ge ce GENRE...
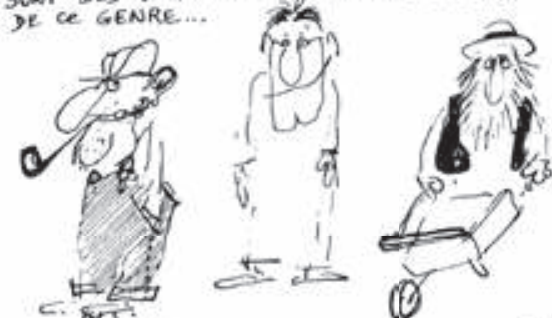

T íst a cause do mecs comme ga qui PEANENT CONSTRURRe DES BARAQWES AUSSi FANTASTiques que Des Tas de GenS ve DUSANT

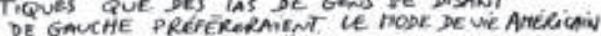
AU mODE DE VIE SOVIETTQUE. 


\section{Comment étudier ces nouveaux objets?}

Le déchet est-il vraiment res nullius, conformément à sa définition juridique? Ou, privé de valeur d'usage et d'échange, se définit-il par une valeur négative? Relative? La définition juridique du déchet, héritée du droit romain, n’est-elle pas obsolète et n'appelle-t-elle pas à être ré-imaginée? De la réduction à la source à la réinvention des modes d'existence de la matière rebut, les enjeux économiques sont toujours présents. Du point de vue des normes et de l'appareillage juridique, du point de vue des formats sociaux et institutionnels qui entourent cet aspect, il y a aussi matière à interroger la notion de «bien» (Douglas \& Isherwood 2007). Comment se valorise et prend consistance ce «bien commun » particulier qu'est le déchet, ce waste common - l'expression est de Ruth Lane, à propos des chiffonniers australiens? Les approches anthropologiques classiques (Thompson 1979, Douglas 2005), constituent un point de départ essentiel pour penser les «pollutions » symboliques mais n'offrent pas les bases adéquates pour étudier les effets des déchets toxiques et nucléaires ou de la pollution atmosphérique sur la santé et le milieu (O’Brien 2011, Le Roux 2011). Les conditions économiques, technologiques ou cosmologiques doivent désormais jouer toutes ensemble dans la catégorisation des restes.

Si l'on cherche à penser la spécificité moderne et urbaine du déchet, il faut aussi nécessairement faire des écarts et interroger les chasseurs-cueilleurs ou les horticulteurs, des sociétés pensées «sans reste» (en tout cas, sans déchet) et pour lesquelles le reste, la scorie, les excreta refoulés prendraient peut-être d'autres formes imaginaires dans un environnement tropical humide caractérisé par la biodégradabilité des objets végétaux - un contexte de nature bien évidemment différent de celui des artéfacts métalliques ou plastiques des civilisations industrielles.

Les restes invitent à penser la valeur d'une façon plus large que celle proposée dans les approches focalisées sur les objets (Myers 2001). Notre démarche inclut non seulement l'emploi (fonctionnel, marchand ou autre) des artéfacts, mais aussi les matières disséminées dans les milieux. Les problèmes les plus importants à ce jour sont peut-être ceux liés aux micro- ou nanoparticules, gaz, lisiers et autres formes matérielles qui sont difficilement caractérisables dans des objets délimités. L'idée de reste nous permet d'englober non seulement des objets, mais aussi des flux de matières en plus des chutes et scories inutilisées. Le reste implique les divers processus, techniques ou naturels, par lesquels les objets se désagrègent; il permet de saisir le «potentiel» (croissance, transformation, devenir) des choses en évitant les limites artificielles d'une pensée articulée autour des seules formes entières et arrêtées. Malgré le rêve d'une économie circulaire vertueuse dans laquelle les objets et matériaux fonctionneraient sans fin, l'idée de reste résiste et permet de voir les choses sous d'autres angles, ceux de la technologie culturelle (Mahias 2004, par exemple) à propos des dimensions économiques, sociales et symboliques de la bouse en Inde, ou celui des potentialités chez Ingold (2012). 


\section{Restes et innovation}

Autant que les rebuts eux-mêmes, la question de leur visibilité est essentielle. Ici s'illustre la place que la société accorde au travail de refoulement, éminemment politique, le négatif, avec lequel l'on doit penser dans le même temps les procédés d'invisibilisation, d'enfouissement, et la valeur accordée au déchet. La langue populaire en témoigne, elle qui a fait dériver la biffe en bifton. Les études contemporaines sur les «travailleurs des déchets», ces acteurs méconnus des sociétés, constituent un champ à part entière recouvrant des catégories, usages, matériaux, façons, organisations sociales très différents dans les pays du Nord ou du Sud (Lhuillier 2005, Jeanjean 2006, Medina 2007, Corteel \& Le Lay 2011, Godard \& Donzel 2014, Ferrell 2005, Nagle 2014, Reno 2016). En plus de la réflexion sur les restes nous avons également souhaité promouvoir des études qui mettent en évidence les dimensions bricolantes et innovantes: les savoirs, savoir-faire, savoir être, que le monde accéléré de la consommation et de la surproduction, appelle en retour et en résistance. Inventeurs, bricoleurs, réparateurs modifient les relations consuméristes aux objets. En les réduisant, les détournant, les réparant, en mutualisant leurs usages ou en économisant leur production, les bricoleurs «s'arrangent avec les moyens du bord », combinent le neuf et le « déjà-là» et créent de nouveaux matériels appropriés à leurs modes de vie et d'action. Ils défendent par leur activité un projet contre entropique (si l'on admet que l'entropie serait la dégradation irréversible de toute chose), où les produits manufacturés sont doués d'une force gestative. Re-faire, c'est donc non seulement réparer et restaurer, mais c'est aussi «faire à nouveau ». Contre l'idée d'« obsolescence programmée» des grandes entreprises, un autre modèle se fait jour dans les petits ateliers du monde, celui d'une «éternité non programmée des techniques».

Les makers, hackers, antiquaires, bricoleurs et vendeurs-acheteurs d'objets d'occasion renvoient à des catégories sociologiques diverses dont le travail et les savoir-faire commencent d'être documentés par les explorateurs des deuxièmes vies d'objets (Anstett \& Ortar 2015). Cela les distingue des études antérieures des biographies ou carrières d'objets (Appadurai 1988, Bromberger \& Chevallier 1999, Debary 2007, Watteau 2011), qui ont exploré les produits, biens et vies d'objets, et peu les processus de transformation. Un certain nombre de travaux Anglo-Saxons récents sur les déchets prônent aussi des approches de type «follow the thing» (Lepawsky \& McNabb 2010, Alexander \& Reno 2012), fort efficaces au demeurant. Nous tentons ici un métissage plus intriqué mêlant les approches de la technologie culturelle (attachées aux matières, objets, processus et connaissances, Cresswell 1996; Lemonnier 2010; Bartholeyns, Joulian \& Govoroff 2010, Gosselain, Zeebrock \& Decroly 2008) à d'autres

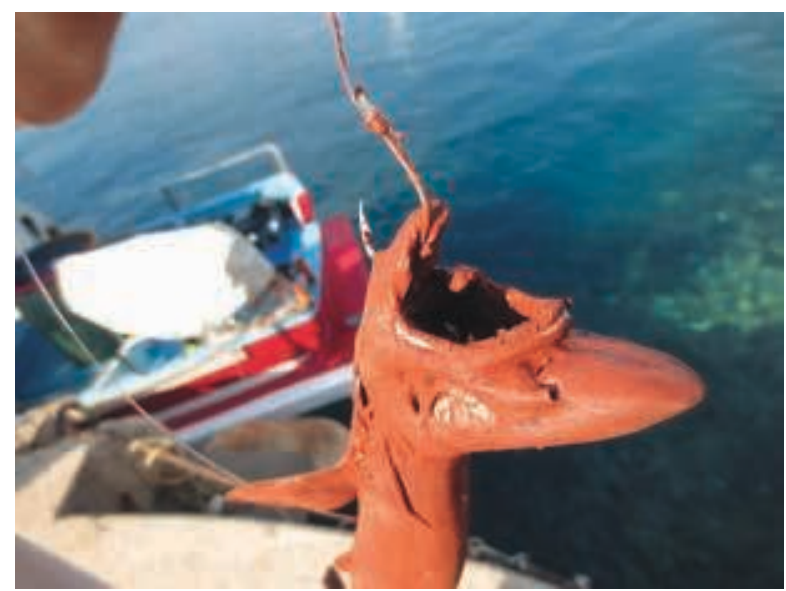

5. «La Gueule ouverte ».
Emblématique du scan-
dale des rejets de boues
rouges dans le Parc natio-
nal des calanques et enjeu
des contradictions entre
les lobbies industriels et
les différents ministères
qui tirent dans des direc-
tions opposées. Celui
de l'industrie gagnant
encore et toujours sur
celui de l'environnement.
Un chien de mer, petit
squale, péché « au »
palangre le 16 juillet 2015
par 300 m de fond dans
le canyon de Cassidaigne. 
plus enclines à aborder les objets en termes de qualité, de performance, d'agence ou de flux et au sein desquelles les matériaux ont conservé une place essentielle (Keane 2005, Revolon et al. 2012, Ingold 2012).

\section{Des creux dans le reste}

En réponse aux trois orientations - la relation entre le reste et les déchets, la question de l'excès et celle des innovations sociales et techniques-, nous avons reçu plus de quatre-vingt-dix propositions d'articles relevant des trois formats: analytique long (en ligne), analytique court, et documentaire, desquels, aidés du comité de lecture, nous avons sélectionné une cinquantaine d’articles, pour la plupart présentés lors du workshop de novembre 2014 ou de séminaires EHESS-MUCEM entre janvier 2014 et juin 2016. Outre le nombre très important de propositions provenant de seize pays différents et intéressant plus de trente aires culturelles distinctes, plus d'une vingtaine d'articles portent sur des questions philosophiques et impliquent l'ensemble de l'économie de la planète (circulation et traitement des déchets électroniques, modèles politiques anticapitalistes, etc.). Nous fûmes donc au premier chef surpris par l'éclectisme et l'originalité des propositions que l'appel avait généré et qui traitaient: des objets des prisonniers du goulag, des processus mémoriels et artéfactuels impliqués dans la cure psychanalytique, des éco-constructions, des enjeux politiques et moraux de la surconsommation, du recyclage d'objets et d'images dans l'art ou le cinéma contemporains, de la question des déchets en littérature, du racisme environnemental aux États-Unis, de la maltraitance animale dans les abattoirs... pour n'en mentionner qu'un mince échantillon. Mais ce qui nous surprit le plus, ce furent certaines absences: les disciplines, domaines et courants théoriques auxquels nous nous attendions et qui ne se retrouvèrent que marginalement, notamment l'archéologie, pour laquelle restes, culture matérielle et mémoire sont les objets centraux et quotidiens d'interrogation. Est-ce que notre approche n'entrait pas dans les catégories actuelles de pensée? Toujours est-il que la «garbage archaeology» nord-américaine (Rathje \& Murphy 2001) qui alliait histoire, sociologie, économie, ethnographie des usages, et introduite dès la fin des années 1970 en France par A. Schnapp (1980) ou J.-P. Demoule (2012), passe aujourd'hui à la trappe ou se voit réinventée par une anthropologie ou une géographie désireuses de s'inscrire au plus près des préoccupations sociales, énergétiques et politiques de l'époque.

Une autre dimension fit aussi étrangement défaut, celle de l'anthropologie et de l'histoire du corps et de ses usages, et plus globalement celle des travaux sur les dimensions culturelles, symboliques et psychologiques liées à la souillure. Il y a encore dix ou quinze ans, de telles perspectives (celle de Douglas dans le monde anglo-saxon; ou en France, celle de Vigarello) auraient été incontournables. Les recherches sur les savoir-faire techniques et sociaux, sur les inventions techniques fondées sur la récupération furent rares et généralement situées dans les pays du Sud. Nous en retînmes un certain nombre dans l'intention de décentrer le regard, de sortir de l'européocentrisme et de le poser, du Sud vers le Nord, ou du Sud vers le Sud, et d'illustrer au mieux les problèmes et dynamiques réellement en jeu. 


\section{Découper et relier}

Les quarante-quatre propositions finalement retenues se distribuent en six ensembles réunissant de façon équilibrée des articles de factures très différentes (réflexions philosophiques, études de terrain, écritures documentaires ou analytiques, locales ou globales). Nous les avons mis en tension en jouant les contrastes plutôt que les réunions thématiques. Il n'y a pas de regroupements « déchets nucléaires» ni d'«économie de la mémoire» ou de « restes et mondialisation », mais des associations de sens qui, nous l'espérons, donnent à l'ouvrage imprimé ou à l'électronique (mâtiné de vidéos, images, BD et données complémentaires) une facture peu habituelle dans la production contemporaine des sciences humaines et sociales.

La structure de «Réparer le monde» s'ouvre sur une première partie «Proliférations» dans laquelle les auteurs abordent la question des restes à toutes les échelles et dans tous les milieux, depuis les satellites, débris par millions tout autour de la planète, jusqu'au continent de plastique dans le Pacifique, en passant par le corps mort ou la question de l'événement traumatique de Fukushima, ou encore par une contribution sur notre difficulté à percevoir ou se représenter les effets et temporalités des résidus nucléaires. Dans la deuxième partie «Bifurcations», nous suivons plusieurs itinéraires d'objets, comment ils se massifient au cours du temps mais aussi comment ils vivent différentes existences, sont détournés, et cohabitent au sein de mondes asynchrones. L'évolutionnisme a fait long feu. En 2016 existent en même temps des sociétés de chasseurs-cueilleurs, agricoles, industrielles ou post-industrielles. Certaines sont des reliques, d'autres résistent, se sauvent peut-être dans un bien commun universel que nous interrogeons depuis la place anthropologique. La troisième partie « Recompositions», face aux contradictions de l'histoire, met les mains dans les matières, les décompose, les recompose, combine et bricole avec d'anciennes filières, les hybride ou invente de nouveaux usages ou de nouvelles façons de faire ensemble. À la dépossession caractérisée des savoirs à laquelle s'associe fréquemment le développement technique moderne, les fablabs répondent activement; nous en donnons divers exemples. La quatrième partie «Requalifications» implique de dépasser les projets et objets, de remettre les hommes et les femmes au centre, de redessiner les formes préétablies du social, de requalifier le travail alternatif ou informel, et aussi de dire la merde, sans détour. Dans la cinquième partie «Politisations», les auteurs creusent les significations politiques des déchets, de l'uranium au patrimoine industriel minier en passant par les ordres et désordres que leurs administrations ont générés. Dans la dernière partie «Refigurations», nous suivons littéralement l'un des auteurs dans son entreprise narrative et anthropologique de donner figure, de re-présenter et se souvenir en représentant les personnes, les ruines, les savoir-faire, les objets déplacés et décontextualisés par nos prédécesseurs. Faut-il rendre les derniers, les détruire, les faire vivre autrement?

Des plasticiens, dessinateurs et photographes amateurs et professionnels nous ont accompagnés tout au long de cette aventure (C. Bosqué, H. Hebig, B. Langmack, K. Maruyama, D. Stier, F. Pourcel, H. Powell). De leurs regards engagés et décalés, ils nous ont permis de quitter les lignes, de lire et donner à voir autrement, plus intensément les hommes et les paysages transformés. Comment vivre de façon optimiste avec les restes du passé dans un monde qui serait, nous dit le web, sans absence et sans manque? Nos efforts de réintroduire l'absence, ou à l'inverse, 
d'éprouver le caillou dans la chaussure, de jouer avec les matières plus qu'avec les objets, de préférer le savoir-faire efficient aux modèles abstraits, les contradictions de la post-modernité (celle qui apparaît virtuelle mais consomme davantage que la modernité industrielle) seront-ils lisibles? Nous l'espérons. De cet ensemble bigarré et bricolé, nous aspirons à ce que le lecteur trouve des matières à penser plus loin et, bien sûr, à faire, plus intensément.

Ajoutons pour finir que même si nous ne l'avons pas ostensiblement affiché, cet ouvrage entre clairement en résistance, l'anthropologie impliquée de Techniques\&Culture se définissant depuis ses débuts dans les années 1970 comme une traduction claire et savante des «modèles du faire» des exclus.

\section{Iconographie}

Image d'ouverture. Dans le marais, à proximité de la centrale de retraitement des eaux usées, de la déchetterie et de l'emplacement forain, les puces demeurent un lieu hors norme où l'économie informelle peut encore exister. Y restent parfois quelques «monstres » intransportables. (C) F. Joulian 2009.

\section{Références}

Alexander, C. \& J. Reno dir. 2012 Economies of Recycling: The Global Transformation of Materials, Values and Social Relations. Londres/New York: Zed Books.

Centre de création industrielle 1984 Déchets: l'art d'accommoder les restes. Catalogue d'exposition. Paris: Centre Georges Pompidou.

Anstett, E. \& N. Ortar dir. 2015 La deuxième vie des objets: Recyclage et récupération dans les sociétés contemporaines. Paris: Pétra.

Appadurai, A. dir. 1988 The Social Life of Things: Commodities in Cultural Perspective. Cambridge: Cambridge University Press.

Barles, S. 2005 L'invention des déchets urbains. France: 17901970. Seyssel: Éditions du Champ Vallon.

Bartholeyns, G., Govoroff, N. \&F. Joulian dir. 2011 Cultures matérielles. Anthologie raisonnée de TechniquesE Culture (2 vols.) TechniquesE Culture 54-55.

Borasi, G., Zardini, M. dir. 2007 Désolé, plus d'essence. L’innovation architecturale en réponse à la crise pétrolière de 1973. Centre Canadien d'Architecture, Montréal. Mantoue: Éditions M. Corraini.

Bromberger, C. \& D. Chevallier dir. 1999 Carrières d'objets. Paris: FMSH Éditions.

Chamak, B. 1997 Le Groupe des dix, ou les avatars des rapports entre science et politique. Monaco : Éditions du Rocher.

1 et 2. (C) F. Joulian 2005, 2008.

3a et b. (C) D. Degner 2014.

4. L'Écologie de Reiser @ Éditions Glénat, 2010.

Reiser et l'énergie solaire en 1970: www.youtube.com/ watch? v=y3zrbGT4Ln0.

5. C Gérard Carrodano, cmca-med.org/film/gerard -carrodano-sentinelle-de-la-mediterranee/.

Corteel, D. \& S. P. Le Lay 2011 Les travailleurs des déchets. Toulouse: Erès.

Cresswell, R. 1996 Prométhée ou Pandore. Propos de technologie culturelle. Paris: Kimé

Debary, O., \& L. Turgeon dir. 2007 Objets et mémoires. Paris: FMSH Éditions / Québec: Université de Laval.

Demoule, J.-P. 2012 «Archéologie, art contemporain et recyclage des déchets », Techniques\&Culture 58 Objets irremplaçables: 160-177.

Douglas, M. 2005 [1966] De la souillure. Paris: La Découverte.

Douglas, M. \& B. Isherwood 2007 [1979] Pour une anthropologie de la consommation. Le monde des biens. Paris: IFM/Regard.

Ferrell, J. 2005 Empire of Scrounge: Inside the Urban Underground of Dumpster Diving, Trash Picking, and Street Scavenging. New York, NYU Press.

Duperrex, M. 2015 Sédiments. www.urbain-trop-urbain. fr/sediments/.

Furniss, J. 2012 Metaphors of Waste: Several Ways of Seeing «Development " and Cairo's Garbage Collectors. Thèse de doctorat, Université d'Oxford.

Gabrys, J. 2013 Digital Rubbish: A Natural History of Electronics. Ann Arbor: University of Michigan Press.

Godard, P. \& A. Donzel 2014 Éboueurs à Marseille. Entre luttes syndicales et pratiques municipales. Préface de Michel Samson. Éditions Syllepse (Le présent avenir). 
Gouhier, J. 1972 Éléments pour une géographie des déchets. Thèse soutenue à l'université de Caen.

Gosselain, O., Zeebroek, R. \&J.-M. Decroly 2008 «Les tribulations d'une casserole chinoise au Niger», Techniques ECulture 51: 18-49.

Guy, H., Jeanjean, A. \& A. Richier 2013 «Le cadavre en procès: une introduction ». TechniquesE Culture $60 \mathrm{Le}$ Cadavre en Procès: 16-29.

Illich, I. 1973 Tools for Conviviality. Londres: Calder \& Boyars. Ingold, T. 2012 « Toward an Ecology of Materials », Annual Review of Anthropology 41 : 427-442.

Irvine, R.D.G. 2014 « Deep Time: An Anthropological Problem », Social Anthropology/Anthropologie Sociale 22 (2): 157-172.

Jeanjean, A. 2006 Basses Fuvres. Une ethnologie du travail dans les égouts. Paris: CTHS.

Joulian, F. \& A. Jeanjean 2013 «Out of culture: la société par ses restes», Annuaire de l'EHESS. Comptes rendus des cours et conférences 2011-2012. Paris: EHESS: 400-402.

Joulian, F., Chevallier, D. \& Y.-P. Tastevin [à paraître en 2017] « Restes et innovation», Annuaire de l'EHESS. Comptes rendus des cours et conférences 2015-2016. Paris: EHESS.

Keane, W. 2005 « Signs are not the Garb of Meaning: On the Social Analysis of Material Things $»$ in D. Miller dir. Materiality. Durham: Duke University Press: 182-205.

Lepawsky, J. \& C. McNabb 2010 «Mapping international flows of electronic waste», The Canadian Geographer 54: 177-195.

Lhuillier, D. 2005 «Le "sale boulot" », Travailler 2005/2 (14): 73-98.

Lemonnier, P. 2011 «L'étude des systèmes techniques, une urgence en technologie culturelle» in G. Bartholeyns, N. Govoroff \& F. Joulian dir. Cultures matérielles. Anthologie raisonnée de TechniquesE Culture, TechniquesECulture 54-55: 46-67.

Le Roux, T. 2011 Le Laboratoire des pollutions industrielles. Paris 1770-1830. Paris: Albin Michel.

Mahias, M.-C. 2004 Le Barattage du monde. Essais d'anthropologie des techniques en Inde. Paris: FMSH Éditions.

Medina, M. 2007 The World's Scavengers: Salvagingfor Sustainable Consumption and Production. Lanham: AltaMira Press.

\section{Pour citer cet article}

Joulian, F., Tastevin, Y.P. \& J. Furniss 2016 « Réparer le monde. Une introduction», TechniquesE Culture 65-66 « Réparer le monde. Excès, reste et innovation », p. 14-27.
Myers, F. dir. 2001 The Empire of Things: Regimes of Value and Material Culture. Oxford: James Currey. Trucks with the Sanitation Workers of New York City. New York: Farrar, Straus and Giroux.

O'Brien, M. 2011 A Crisis of Waste? Understanding the Rubbish Society. London: Routledge.

Rathje, W. 1980 «Lopération poubelle. Une nouvelle façon de regarder les problèmes de l'archéologie» in A. Schnapp dir. L'Archéologie aujourd'hui. Paris: Hachette: 251-262.

Rathje, W. \& C. Murphy 2001 [1992] Rubbish! The Archaeology of Garbage. Arizona Press University.

Reiser, J.-M. 1995 Sont pas plus forts que nous. Les années Reiser 1975. Paris: Albin Michel.

Reno, J. 2016 Waste Away: Working and Living with a North American Landfill. Oakland, California: University of California Press.

Revolon, S., Lemonnier, P. \& M. Bailly dir. 2012 Techniques ECulture 58 Objets irremplaçables.

Schnapp, A. dir. 1980 L'Archéologie aujourd'hui. Paris: Hachette.

Schumacher, E.-F. 1973 Small is beautiful: A Study of Economics as if People Mattered. Londres: Vintage Books.

Steffen, W. et al. 2011 «The Anthropocene: From Global Change to Planetary Stewardship », Ambio 40: 739-761.

Strasser, S. 2000 Waste and Want: A Social History of Trash. New York: Henry Holt \& Co.

Tastevin, Y.-P. 2012 Autorickshaw: Émergence et recompositions d'une filière entre l'Inde, l'Égypte et le Congo. Université de Paris Ouest Nanterre La Défense.

Thompson, M. 1979 Rubbish Theory: The Creation and Destruction of Value. Oxford: Oxford University Press.

Vigarello, G. 1985 Le propre et le sale. L'hygiène du corps depuis le Moyen Âge. Paris: Éditions du Seuil.

Watteau, F. dir. 2011 Profils d'objets. Approches d'anthropologues et d'archéologues. Paris: De Boccard.

Zalasiewicz, J. et al. 2012 «The Anthropocene: a new epoch of geological time? », Philosophical Transactions of the Royal Society 369: 835-841.

Zimring, C. 2005 Cash for Your Trash: Scrap Recycling in America. London: Rutgers University Press.
Nagle, R. 2014 Picking Up: On the Streets and Behind the

. Excès, reste et innovation $r, 14-27$. 